\title{
Arbeitszeitgestaltung in der digitalisierten Arbeitswelt: Ein systematisches Literatur Review zur Wirkung von Arbeitszeitverkürzung in Bezug auf die psychische Gesundheit
}

\author{
Virgilia V. Jansen-Preilowski · Agnieszka Paruzel ${ }^{1}$ · Günter W. Maier ${ }^{1}$
}

Online publiziert: 11 . August 2020

(c) Der/die Autor(en) 2020

\section{Zusammenfassung}

Dieser Beitrag der Zeitschrift Gruppe. Interaktion. Organisation. (GIO) stellt ein systematisches Review zum Zusammenhang der Arbeitszeitverkürzung (bei vollem Gehalt) und psychischen Gesundheit auf der Grundlage vorhandener Studien vor, um Chancen und Risiken besser einschätzen und beurteilen zu können. Die Diskussion über die Verkürzung von Arbeitszeiten wird nicht zuletzt durch die vielfältigen Möglichkeiten der Digitalisierung der Arbeit verstärkt. Die Literatursuche ergab insgesamt 114 Artikel, wovon drei qualitative, neun quantitative Studien und eine Mixed Method Studie in die Analyse aufgenommen wurden. Aus den Ergebnissen wurde deutlich, dass eine verkürzte Arbeitszeit bei gleichbleibendem Lohn zu einer Verringerung von arbeitsbedingten Belastungen bei Beschäftigten beitragen. Die Umsetzung der Verkürzung erfolgte auf drei unterschiedliche Weisen: I. ohne Veränderung der Arbeitsprozesse, II. ohne Veränderung der Arbeitsprozesse, jedoch mit zusätzlichen Beschäftigten oder III. mit einer vorhergehenden Veränderung der Arbeitsprozesse, ohne zusätzliche Angestellte. Zwar gibt es bis dato noch keine allgemeingültige Empfehlung für die Umsetzung in die Praxis, allerdings wird für eine erfolgreiche Umsetzung empfohlen, die Reduktion der Arbeitszeit vorab zu planen und Optimierungsmöglichkeiten für die Ausführung von Tätigkeiten und Arbeitsprozessen zu identifizieren (III.).

Schlüsselwörter Arbeitszeit · Arbeitszeitverkürzung · Digitalisierung · Psychische Gesundheit · Stress · Wohlbefinden · Erholung

\section{Organization of working time in digitized work environments: a systematic literature review on the effects of reduced working hours on mental health}

\begin{abstract}
In this systematic literature review in the special edition "Digitalisierte Arbeitswelt" of the journal "Gruppe. Interaktion. Organisation. (GIO)" we analyzed the relationship between worktime reduction and psychological health. A total of 114 papers were identified and three qualitative, nine quantitative studies and one mixed method studies were included. It was found that a shortening in working time with remaining salary can have a positive influence on work stressors and work related stress reactions. In the primary studies were three different implementation categories of the worktime reduction identified: I. without any changes in processes, II. no changes in processes combined with additional employees or III. changes in processes without additional employees. Yet, there is no general overall implementation strategy, we suggest organizations/employers to organize the shortening of working hours and to identify possibilities to optimize tasks and processes beforehand (III.).
\end{abstract}

Keywords Working hours · Working time reduction · Digitalization · Psychological health · Stress · Well-being · Recovery

Virgilia V. Jansen-Preilowski

virgilia.jansen-preilowski@uni-bielefeld.de

1 Abteilung für Psychologie, Universität Bielefeld, Postfach

100131, 33501 Bielefeld, Deutschland 


\section{Einleitung}

„Wollt ihr weniger arbeiten und genauso viel verdienen?““ (Die Zeit, Jacobs 2018), ,5-Hour Workdays? 4-Day Workweeks? Yes, Please“ (New York Times, Newport 2019), "Should we rethink our working hours to boost productivity?“ (Personnel Today, Webber 2019) oder „Kürzer, besser glücklicher" (Frankfurter Allgemeine Zeitung, Bärschneider 2020) - das sind nur einige aktuelle Schlagzeilen aus weltweiten Zeitungsberichten, in denen auf ein neues Arbeitszeitmodell der sogenannten New Work Bewegung aufmerksam gemacht wird. Bei diesem Arbeitszeitmodell geht es darum, bei gleichbleibendem Gehalt die Arbeitszeit (substanziell) zu reduzieren. Kleine (bspw. Rheingans Digital Enabler) und große (bspw. Microsoft) Organisationen, in unterschiedlichen Branchen (z.B. IT, Sozial- oder Gesundheitswesen) und Ländern (z.B. Deutschland, Schweden oder USA) erprobten oder erproben dieses neue Modell, ja einige haben es sogar schon wieder zurückgenommen (Stern, Grimm 2017). In den Artikeln werden positive Erfahrungen angesprochen - wie etwa verbesserte Erholung oder geringeres Stressempfinden, aber auch mutmaßliche Nachteile erörtert - wie geringer sozialer Zusammenhalt im Unternehmen oder höhere Betriebsausgaben durch mehr Personal. Die Intensität der Diskussion über solche Ansätze der Arbeitszeitgestaltung ist inzwischen so weit gediehen, dass selbst die Presse Fake-Meldungen über die angeblichen Pläne der finnischen Regierungschefin europa- und weltweit verbreitet (MacDougall 2020). Andererseits ist der Erkenntnisstand zur Wirkung dieses Modells gegenwärtig noch recht gering. Daher ist es das Ziel dieses Reviews, die gegenwärtig vorliegenden Befunde systematisch zu erschließen und zusammenfassend zu bewerten. Dazu werden wir zunächst darstellen, wie sich dieses Modell in die historische Entwicklung der Arbeitszeit einfügen lässt, um anschließend die theoretischen Grundlagen über die mögliche Wirkung dieser Form der Arbeitszeitreduktion zu erörtern. Darauf aufbauend stellen wir die Ergebnisse des systematischen Reviews aus bisherigen Einzelfallberichten sowie ersten quantitativen Studien dar. Abschließend ziehen wir ein Resümee und diskutieren, inwieweit diese Erfahrungen über diese spezielle Form der Arbeitszeitgestaltung zu mehr Erkenntnissen über die Wirkung von Arbeit führen kann.

Historisch gesehen fügt sich die aktuelle Auseinandersetzung um diese spezielle Form der Arbeitszeitverkürzung in den generellen Trend der Arbeitszeitveränderung in den letzten 150 Jahren ein. Anfang des 20. Jahrhunderts wurde im Zeitalter der Industrialisierung die Arbeit durch einen immer höheren Automatisierungsgrad um ein Vielfaches erleichtert, wodurch sich die Produktionszeit verkürzte (Anttila 2005). Auf Anraten des Arbeitswissenschaftlers Frederick Taylor änderte Henry Ford in seinem Unternehmen die Arbeitszeit von neun auf acht Stunden täglich und von sechs auf fünf Tage pro Woche. Gleichzeitig verdoppelte Ford den Stundenlohn seiner Mitarbeitenden. Die Befürchtung von anderen, er würde damit seiner Firma schaden, bestätigte sich nicht. Die Produktivität der Beschäftigten steigerte sich trotz verkürzter Arbeitszeit. Im Zuge der fortschreitenden Digitalisierung stellt sich immer wieder die Frage, wie sich Arbeitsbedingungen, unter anderem auch Arbeitszeiten, für Beschäftigte entwickeln werden. In den vergangenen Jahrzehnten haben Technologien der Digitalisierung in Unternehmen effizientere Arbeitsabläufe ermöglicht und die Produktivität sowie Unternehmensleistung gesteigert (Luftman et al. 2017; Ashford und Kallis 2013). Produktivitätssteigerungen stellten sich nicht nur wegen der Effizienz neuer Technologien ein, sondern wurden auch durch Substituierung des Personals durch Maschinen erreicht (Ashford und Kallis 2013). Ein Trend, den Frey und Osborne (2017) auch für die zukünftige Entwicklung prognostizieren, denn ihnen zufolge sollen in den USA $47 \%$ der Jobs möglicherweise automatisiert werden. Eine genaue Übertragbarkeit der Prognosen auf die EU Länder gestaltet sich aufgrund unterschiedlicher Ausbildungsberufe schwierig (Diewald et al. 2020). Bei der Analyse von Studien zu Substituierbarkeitsprojektionen für Deutschland und die EU, erfassten Diewald et al. (2020) Prognosen, die zwischen $12-51 \%$ der Jobs in Deutschland durch Computerisierung als bedroht sehen.

Trotz der vermeintlichen Zunahme an Arbeitseffizienz scheint in den letzten Jahren aber auch die Beanspruchung der Beschäftigten zugenommen zu haben. Aus dem Stressreport (Lohmann-Haislah 2012) der Bundesanstalt für Arbeitsschutz und Arbeitsmedizin (BAuA) geht hervor, dass die Ausdehnung der Arbeitsstunden mit erhöhter wahrgenommener psychischer Belastung im Zusammenhang steht. Laut einer Befragung der BAuA zur Arbeitszeit, arbeiten Vollzeitbeschäftigte in Deutschland durchschnittlich $43,4 \mathrm{~h}$ pro Woche (Bundesanstalt für Arbeitsschutz und Arbeitsmedizin, Wöhrmann et al. 2016). Abhängig vom Tarifvertrag sind dies ca. fünf Stunden pro Woche länger als vertraglich $(38,6 \mathrm{~h})$ vereinbart und somit Überstunden. Ebenfalls geht aus dem Bericht hervor, dass $55 \%$ der Vollzeiterwerbstätigen gerne ihre wöchentliche Arbeitszeit um zehn Stunden reduzieren würden. Auch in anderen Ländern scheinen viele Angestellte länger als vertraglich geregelt zu überarbeiten. In den EU-28 Ländern arbeiteten $15 \%$ der Arbeitnehmenden im Jahr 2015 mehr als $48 \mathrm{~h}$ pro Woche (ParentThirion et al. 2016).

Eine Möglichkeit dieser Belastung zu entgegen, könnte die Arbeitszeitverkürzung bei gleichbleibendem Lohn darstellen. Einige Arbeitgeber setzen den Wunsch der Angestellten nach mehr Freizeit bereits um, jedoch teilweise mit Einkommenseinbußen. Beispielsweise erhalten Beschäftigte der Deutschen Bahn nun die Möglichkeit zwischen einem höheren Lohn oder mehr Freizeit zu wählen (DB, Kaiser 
2018). Auch die Gewerkschaft IG Metall hat vor kurzer Zeit eine (zeitweise) Reduzierung der Wochenarbeitszeit durchgesetzt, sodass Beschäftigte nun über eine Dauer von zwei Jahren, mit einer Minderung ihres Gehalts, ihre Arbeitszeit auf $28 \mathrm{~h}$ pro Woche reduzieren können (Böckmann 2018). Ein geringeres Einkommen könnte allerdings einen weiteren Stressor für viele Beschäftigte darstellen. Einzelne Unternehmen setzen AZV bereits bei vollem Lohnausgleich um und geben an, trotzdem eine gesteigerte Produktivität zu erreichen. Rheingans Digital Enabler hat zum Beispiel vor mehr als zwei Jahren ihre Arbeitszeit auf $25 \mathrm{~h}$, verteilt auf fünf Tagen pro Woche, verringert (FAZ 2019). Die Mitarbeitenden berichten, dass sie sich trotz gesteigerter Produktivität erholter fühlen, sowie kreativer und konzentrierter arbeiten können.

Um den Stand der Forschung zu dokumentieren und Empfehlungen für die Umsetzung in Organisationen abzuleiten, untersuchen wir, inwiefern eine Arbeitszeitverkürzung bei gleichbleibendem Lohn auf die psychische Gesundheit wirkt. Dazu sollen die wissenschaftlichen Erkenntnisse erstmals in einem systematischen Literatur Review analysiert und zusammengefasst werden. Dabei folgen wir den Empfehlungen von Davis et al. (2014) und berücksichtigen bei der Analyse sowohl qualitative als auch quantitative Studien, was einen Vorteil gegenüber einer Metaanalyse darstellt.

\subsection{Wirkung von Arbeitszeit}

Lange Arbeitszeiten stehen im Zusammenhang mit psychischer und physischer Gesundheit (Barnett 2006). In der Metaanalyse von Sparks et al. (1997) konnte ein signifikanter positiver Zusammenhang von Arbeitsstunden und Krankheitssymptomen gezeigt werden, was darauf hinweist, dass lange Arbeitszeiten schädlich für die Gesundheit sein können. Im Überblick zur Wirkung von Arbeitsstunden zeigen erste Reviews, welche Faktoren im Zusammenhang mit dem Umfang von Arbeitszeit stehen (Bannai und Tamakoshi 2014; Solovieva et al. 2013; Kang et al. 2012). In einem Review wurde herausgestellt, dass der Einfluss von der Anzahl der Arbeitsstunden auf den Gesundheitszustand nicht direkt verläuft (Ganster et al. 2018). Stattdessen wird vermutet, dass der Zusammenhang zwischen langen Arbeitsstunden und der Gesundheit (Stressreaktionen, Wohl-

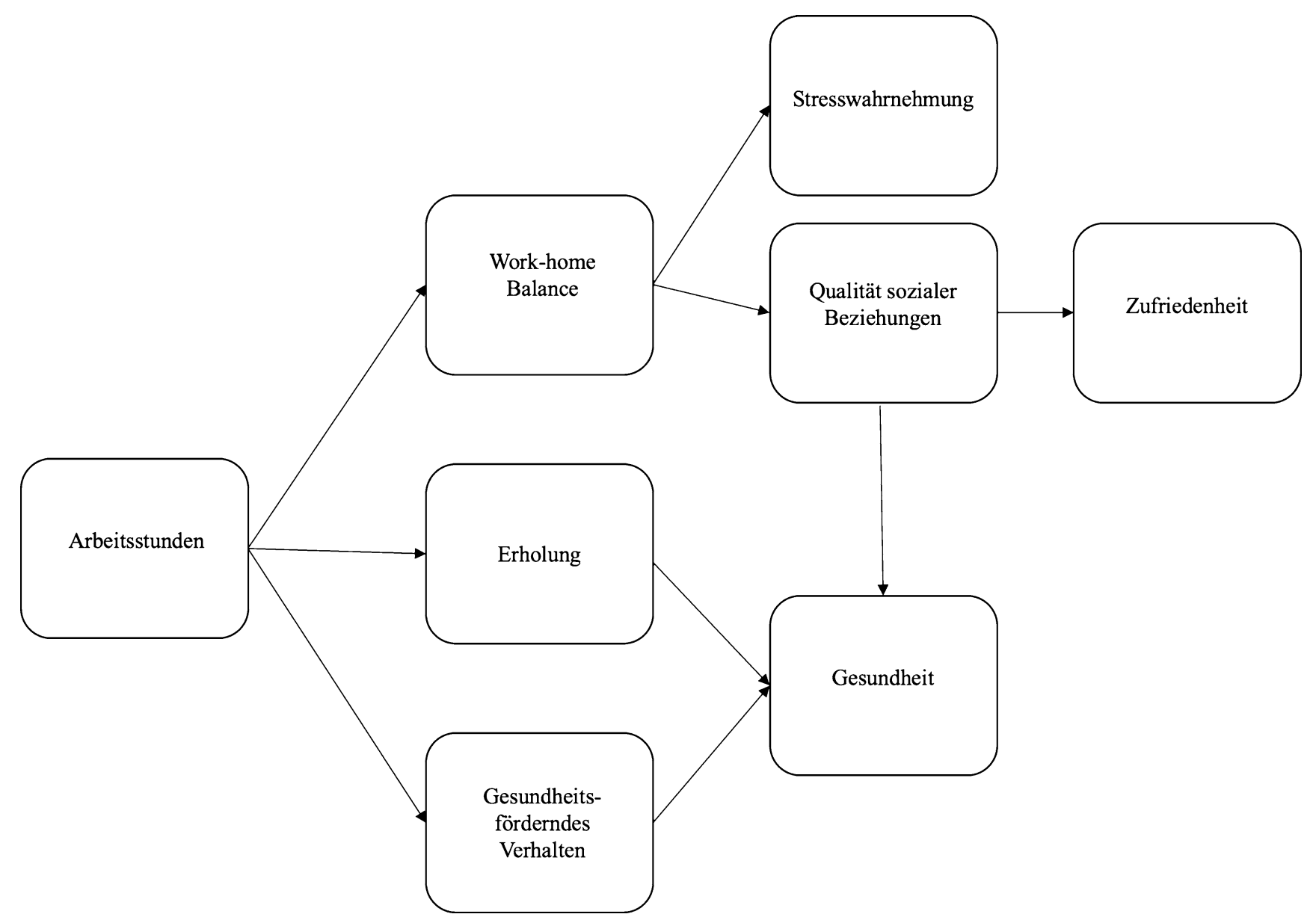

Abb. 1 Mediationsmodell zu Arbeitsstunden nach Ganster et al. (2018) 
befinden, psychische und physische Gesundheit) über drei der folgenden Mediatoren verläuft: Erholung von Erschöpfungserscheinungen, Vereinbarkeit von Familie und Beruf (work-home Balance) sowie gesundheitsförderndes Verhalten von Beschäftigten (Abb. 1).

Studien aus der Organisationspsychologie und verwandten Feldern haben die Erholung von Arbeit als wichtigen Mechanismus identifiziert, der erklärt wie Mitarbeitende trotz hohen Arbeitsanforderungen energetisch, motiviert und gesund bleiben können (Sonnentag et al. 2010; Trougakos et al. 2008). Dies lässt sich mit dem EffortRecovery Modell (Meijman und Mulder 1998) erklären. Diesem Modell zufolge wird nach einer hohen Beanspruchung eine ausreichende Erholung benötigt, um gesund zu bleiben. Eine dauerhafte komprimierte Erholung stellt eine hohe Beanspruchung dar und führt zu langfristigen Stressreaktionen und somit zu chronischen psychischen und physischen Erkrankungen. Erholung wird dabei als zirkulärer Prozess definiert, bei dem keine hohe Beanspruchung vorliegt, um physische oder psychische Belastungssymptome, die durch Arbeitsanforderungen oder stressvolle Ereignisse verursacht wurden, zu verringern oder zu eliminieren. Erholung findet vornehmlich in der Freizeit statt und ist somit essentiell für individuelles Wohlbefinden (Sonnentag 2001). Bei einer Verkürzung der Arbeitszeit könnte womöglich zum einen die Anstrengung verringert und zum anderen die Möglichkeit der Erholung durch mehr Freizeit erhöht werden und sich somit positiv auf die Gesundheit und das Stressempfinden auswirken. Somit hätten Beschäftigte auch mehr Zeit für soziale Aktivitäten und eine verbesserte Work-Life Balance.

Mögliche Erklärungen in Bezug auf die Machbarkeit der AZV liefern Studien zu Leerlaufzeiten (idle time) und das Parkinson'sche Gesetz (Parkinson und Osborn 1957). Diese Theorie geht davon aus, dass Tätigkeiten an die verfügbare Arbeitszeit angepasst werden. Das bedeutet, dass bei einer gleichbleibenden Anzahl von Aufgaben die Dauer der Erledigung auf die vorgegebene Zeit entweder ausgedehnt oder verkürzt wird. In Branchen, wo Leerlaufzeiten zu verzeichnen sind, könnten bei einer AZV Tätigkeiten effizienter ausgeführt werden, was sich wiederum positiv auf die Motivation der Beschäftigten auswirken könnte (Brodsky und Amabile 2018).

\subsection{Mögliche negative Seiten der AZV}

Von einer AZV sind aber nicht nur positive Folgen zu erwarten, sondern auch eine Reihe negativer. Dazu zählen die Folgen für die Intensität der Arbeit, die inhaltliche und soziale Qualität der Arbeit, sowie kontinuierliche Erholung durch Mikropausen während der Arbeit. Durch eine AZV kann es zu einer zu hohen Intensität der Arbeit (Verdichtung) kommen und somit kann AZV zu Stressreaktionen führen (De Spiegelaere und Piasna 2017), vor allem, wenn die Arbeit zuvor in ihren Abläufen nicht optimiert, sondern lediglich die Stundenanzahl reduziert wurde. Dabei bestünde das Risiko, dass Aufgaben in der vorgegebenen Zeit nicht zu erledigen sind. Dadurch würden Beschäftigte vermehrt zu Hause arbeiten und in ihrer Freizeit erreichbar sein, womit das Risiko für eine Überlastung bestünde (Thörel et al. 2020). Bei der Optimierung von Arbeit könnte die Reduzierung der Komplexität, z.B. durch technische Hilfsmittel, die psychische Qualität von inhaltlichen Anforderungen abnehmen und somit zu einer Verringerung der Aufgabenattraktivität und der intrinsischen Motivation führen (Hackman und Oldham 1976). Einen weiteren möglichen negativen Aspekt stellt die verringerte Zeit an beruflichen sozialen Austausch dar, wodurch das Grundbedürfnis nach sozialer Eingebundenheit gefährdet sein könnte (Deci und Ryan 2008). Dies könnte zu einer Verschlechterung von Teamarbeit bis hin zu Konflikten führen. Bei der Verkürzung mit einhergehender Verdichtung von Arbeit könnten Mikropausen während kurzer Leerläufe in Prozesse wegfallen, wodurch die Möglichkeiten zur Erholung während der Arbeitszeit reduziert werden würden (Schaff 2019).

Es scheint widersprüchliche Effekte von der Wirkung von AZV zu geben, die noch nicht systematisch analysiert wurden. Zusätzlich wird in einigen Studien zu Arbeitszeit eine Verkürzung der Arbeitszeit empfohlen, um die psychische Gesundheit von Beschäftigten und Möglichkeiten zur Erholung zu fördern (Barck-Holst et al. 2019; Schiller et al. 2017; Schiller et al. 2018). Aufgrund des geringen Erkenntnisstands stellt sich die Frage, welchen Effekt AZV auf die psychische Gesundheit hat. Zur systematischen Analyse nutzen wir als theoretisch angenommenes Wirkmodell das Mediationsmodell von Ganster et al. (2018) welches aus dem Review zu Arbeitsstunden hervorgeht (vgl. Abb. 1). Aus den genannten Mediatoren (Workhome Balance, Erholung und gesundheitsförderndes Verhalten) und den abhängigen Variablen (psychische Gesundheit und Stresswahrnehmung) wird abgeleitet, welche Ergebnisse aus den Primärstudien exzerpiert werden.

\section{Methoden}

\subsection{Suchstrategie}

Es wurde eine systematische Literaturrecherche in den Datenbanken PsycINFO und Web of Science durchgeführt sowie in Referenzlisten und gezielte nach einschlägigen Autoren gesucht. Da es bisher in der Literatur keinen einheitlichen Begriff zur AZV gibt, wurden mehrere Begriffe in die Suche eingeschlossen. Die Suche wurde am 20. Juli 2019 durchgeführt und am 5. März 2020 aktualisiert. Als abhängige Variablen wurden auf Basis eines 
Reviews zu den Auswirkungen langer Arbeitszeiten auf die Gesundheit (Ganster et al. 2018) psychische Gesundheit und Stresswahrnehmung ausgewählt. Zusätzlich wurden die vermuteten Mediatoren für diesen Zusammenhang aufgenommen (work-home Balance, Erholung sowie gesundheitsförderndes Verhalten). Zur Recherche diente der folgende Suchterm: (("reduction in working hours" OR "reduced working time" OR "reduced work time" OR "worktime reduction" OR "working time reduction" OR "reduced working hours") AND ("stress" OR "well-being" OR "health" OR "psychological health" OR "healthy behavior" OR "recovery" OR "work-home balance" OR "social relationships" OR "satisfaction")). Um deutschsprachige Literatur mit einzuschließen, wurde der folgende Suchterm eingesetzt: (("Arbeitszeitverkürzung" OR "Reduzierung der Arbeitszeit" OR "verkürzte Arbeitszeit" OR "Verringerung der Arbeitszeit") AND ("Stress" OR "Wohlbefinden" OR "Gesundheit" OR "psychische Gesundheit" OR "gesundheitsförderndes Verhalten" OR "Erholung" OR "work-home Balance" OR "soziale Beziehungen" OR "Zufriedenheit")). Zudem wurden die Referenzlisten der Artikel nach weiteren relevanten Studien durchsucht.

\subsection{Auswahlkriterien}

\subsubsection{Unabhängige Variable AZV}

Voraussetzung war zum einen, dass die AZV bei gleichbleibendem Lohn implementiert wurde, sodass eine Veränderung des Gehalts keinen Einfluss spielte. Der Umfang der AZV stellte bei der Auswahl kein Kriterium dar. Zum anderen handelte es sich bei der Reduktion um keine Teilzeitbeschäftigung, Kurzarbeit oder Altersteilzeit, um weitere Stressoren, wie zum Beispiel Einkommenseinbußen oder Furcht vor Arbeitslosigkeit auszuschließen. Daher ist der volle Lohnausgleich bei AZV ein wesentlicher Unterschied zu anderen Teilzeitmodellen.

\subsubsection{Abhängige Variablen}

Die abhängigen Variablen wurden auf Basis eines Reviews zu Zusammenhängen langer Arbeitszeit mit Gesundheit abgeleitet (Ganster et al. 2018). Es wurden Studien inkludiert, die mindestens eine der folgenden abhängigen Variablen zum Oberbegriff Gesundheit beinhalteten: work-home Balance, Erholung, gesundheitsförderndes Verhalten Stress, psychische Gesundheit oder Stresswahrnehmung.

\subsubsection{Design der Primärstudien}

Bei der Analyse wurden Davis et al. (2014) zufolge Studien sowohl mit quantitativen als auch mit qualitativem For-

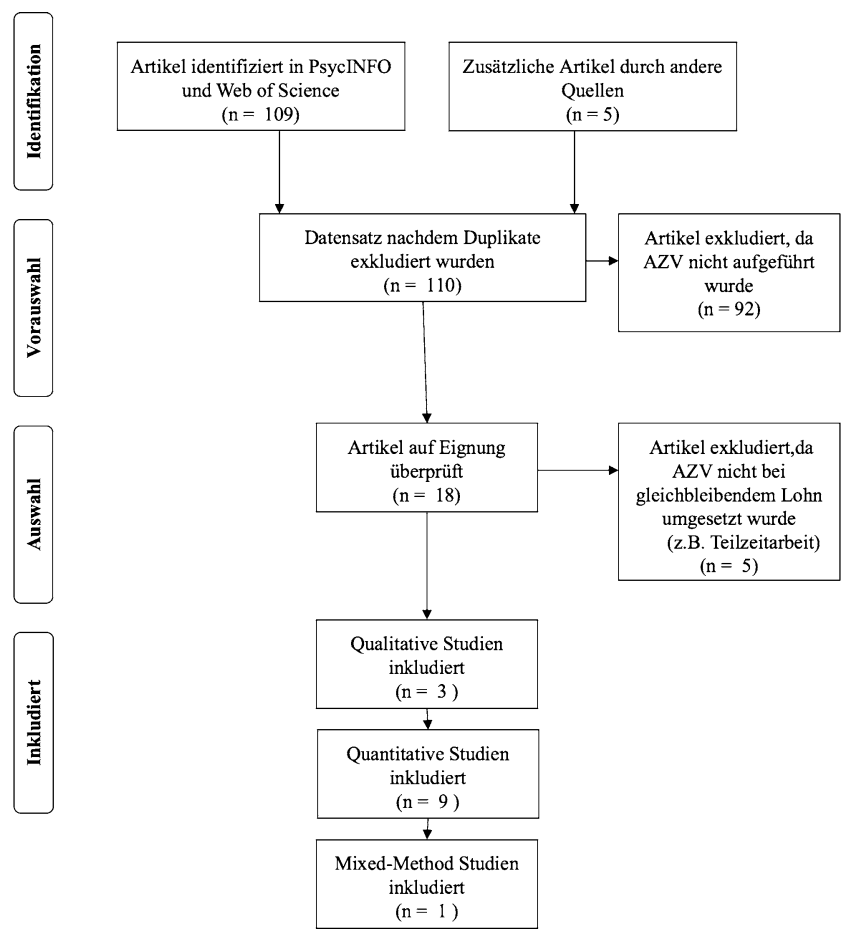

Abb. 2 Flowchart systematische Literatursuche

schungsdesign berücksichtigt. Aus den Primärstudien wurden aus den Ergebnissen die folgenden Faktoren exzerpiert: Work-home Balance, Erholung, gesundheitsförderndes Verhalten, psychische Gesundheit und Stressempfinden. Zur vollständigen Beschreibung der Befunde wurden ebenfalls Kontextvariablen (Branche und Land) sowie methodische Angaben (Untersuchungsdesign) exzerpiert.

\section{Ergebnisse}

Das Ergebnis unserer systematischen Literatursuche wird in Abb. 2 dargestellt. Für das Flussdiagramm haben wir die Prisma Statement (Moher et al. 2009) Vorlage verwendet. Insgesamt wurden mit der Suchstrategie in beiden Datenbanken 114 Artikel angezeigt. Fünf davon wurden doppelt aufgeführt. Ein großer Teil von Artikeln $(n=92)$ musste ausgeschlossen werden, da AZV nicht umgesetzt wurde. Weitere Artikel $(n=5)$ mussten ausgeschlossen werden, weil die AZV nicht bei gleichbleibendem Lohn durchgeführt wurde (z.B. Teilzeitarbeit). So konnten abschließend 13 Publikationen in das Review aufgenommen werden., wovon drei qualitative, neun quantitative und eine Mixed Method Studie waren.

In Tab. 1 wird die Übersicht der Ergebnisse dargestellt. Es wurden die Stichprobengröße $(N)$, die Nationalität der Stichprobe, die Branche der Beschäftigten, das Design der Studien, die Umsetzung der unabhängigen Variable (Umset- 


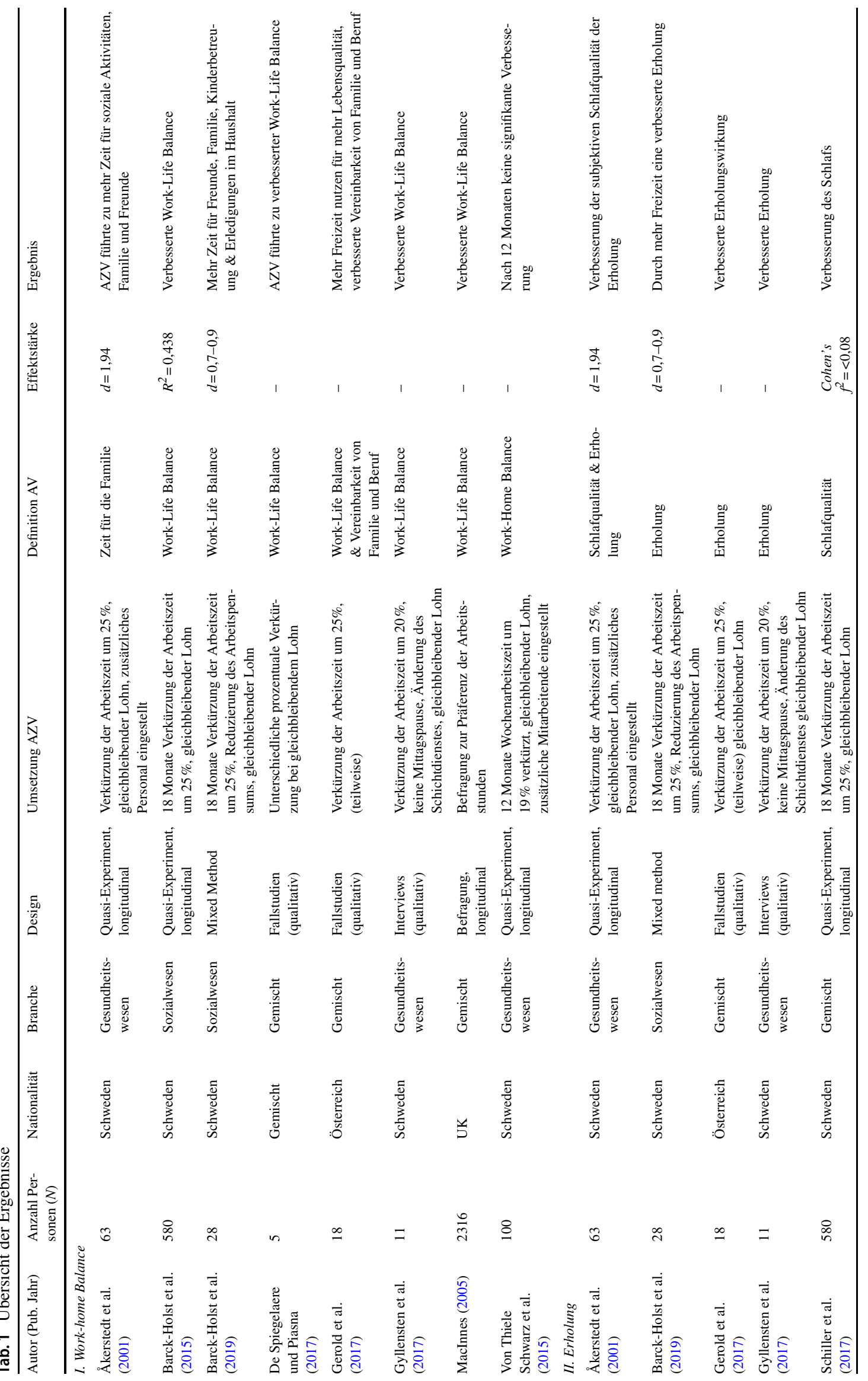




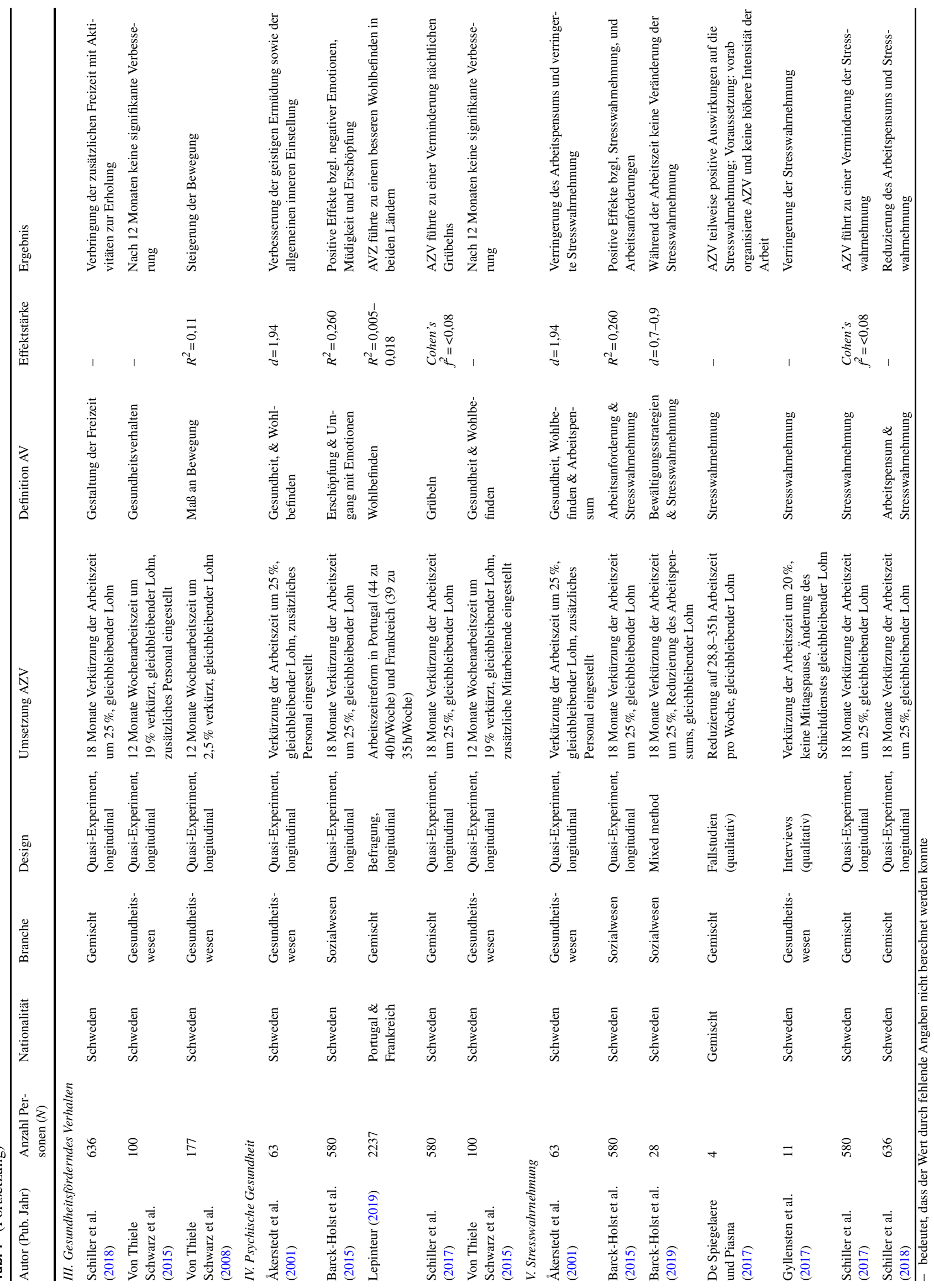


Abb. 3 Darstellung der Häufigkeiten der gemessenen Kriterien in $\%$

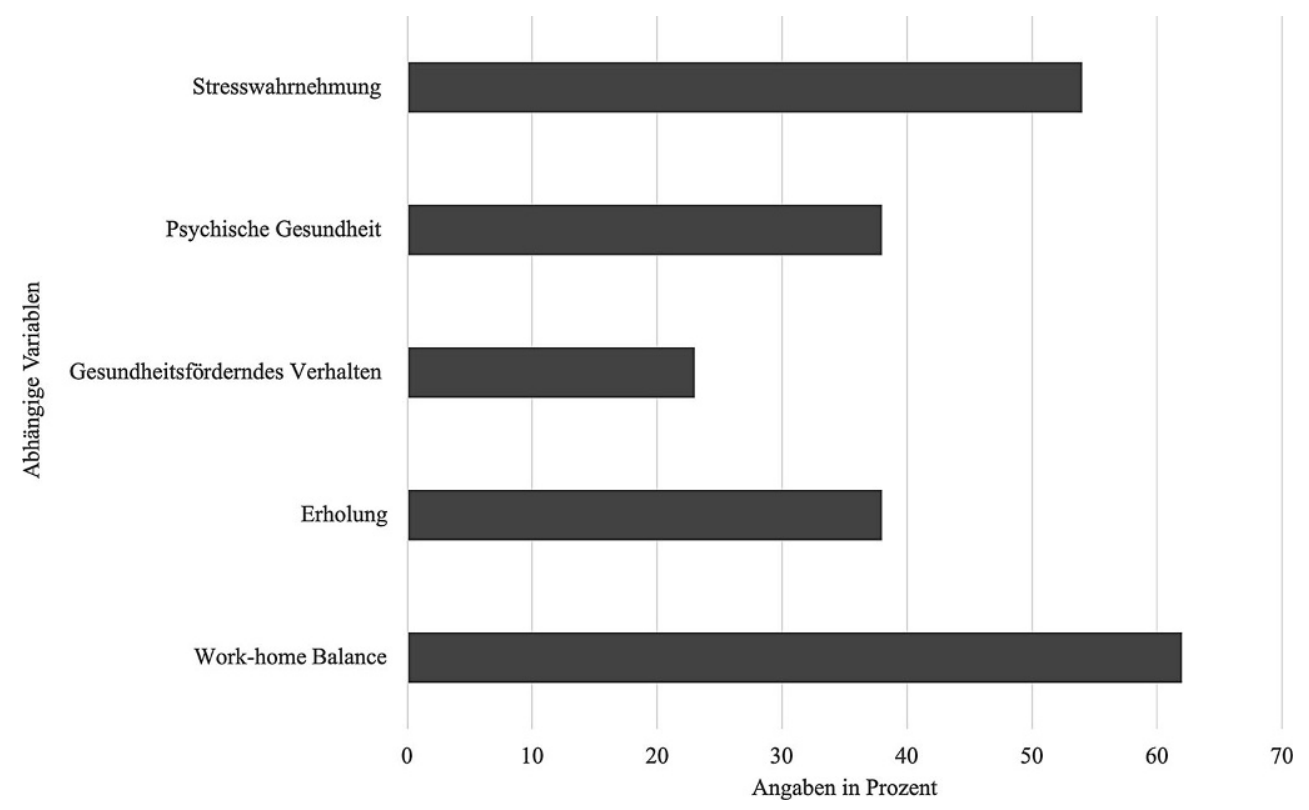

zung $A Z V)$, die Definition der abhängigen Variablen $(A V)$, die Effektstärke, sowie das Ergebnis analysiert.

\subsection{Umsetzung von AZV}

In den Primärstudien wurde AZV bei gleichbleibendem Lohn unterschiedlich umgesetzt (Tab. 1). Die Reduktion der Arbeitsstunden betrug zwischen 2,5-25\%. Dabei variierte die Verkürzung entweder in der Verteilung auf die Arbeitstage pro Woche (von fünf auf vier Tage) oder in der Anzahl der Arbeitsstunden pro Tag. Zudem sind drei unterschiedliche Kategorien in der Umsetzung in die Praxis zu erkennen, jedoch wurde die genaue Umsetzung nicht in allen Studien angegeben:

I. AZV ohne Veränderung der Arbeitsprozesse bzw. des -pensums

II. AZV ohne Veränderung der Arbeitsprozesse, allerdings in Kombination mit zusätzlichem Personal

III. AZV mit einer vorhergehenden Veränderung der Arbeitsprozesse, ohne zusätzliches Personal

\subsection{Methodische Aspekte}

Die Mehrheit der Studien hatte ein quasi-experimentelles Design. Bei $69 \%$ der gesamten Studien handelte es sich um Längsschnittstudien mit mehreren Messzeitpunkten. Die Stichproben waren zwar internationaler Herkunft, allerdings wurde mehr als die Hälfte $(62 \%)$ der Studien in Schweden und $31 \%$ im Gesundheitswesen erhoben. Insgesamt ging eine Stichprobengröße von $N=6750$ zur Analyse in das Review ein. Aus den Studien von de Spiegelaere und Piasna (2017) wurden vier von fünf Fallstudien be- rücksichtigt, da eine Studie eine landesweite AZV in den Niederlanden mit Lohnkürzungen einherging und daher nicht für unsere Analyse geeignet war.

\subsection{Ergebnisse der Studien}

Die Ergebnisse wurden in Tab. 1 in fünf Kategorien der abhängigen Variablen unterteilt: Work-home Balance (I.), Erholung (II.), gesundheitsförderndes Verhalten (III.), psychische Gesundheit (IV.) sowie Stresswahrnehmung (V.). Die Häufigkeit der Messung der aufgenommenen Kriterien in den 13 analysierten Studien war wie folgt (vgl. Abb. 3): Work-home Balance (62\%), Erholung (38\%), gesundheitsförderndes Verhalten (23\%), psychische Gesundheit (38\%) sowie Stresswahrnehmung (54\%). Innerhalb der Kategorien wurden die Ergebnisse alphabetisch nach Autoren sortiert. Sofern Studien Ergebnisse aus mehreren Kategorien verzeichneten, wurden diese mehrfach aufgeführt. Da bei einzelnen Ergebnissen keine Effektstärke angegeben war, wurde bei vorhandenen Angaben diese berechnet. Dabei zeigte die AVZ bei einem Großteil der Studien (92\%) positive Auswirkungen. Lediglich eine Studie konnte nach zwölf Monaten weder einen positiven noch einen negativen Zusammenhang feststellen. Von Thiele Schwarz et al. (2015) erklärten dies mit einer möglichen nicht gut umgesetzten Durchführung von AZV. Eine Zusammenfassung der Ergebnisse wurde in Abb. 4 dargestellt.

\subsubsection{Work-home Balance}

Bei sieben von acht Studien führte die AZV zu einer Verbesserung der Work-home Balance. Dabei führte der Anstieg der Freizeit zu mehr Zeit für soziale Aktivitäten sowie 


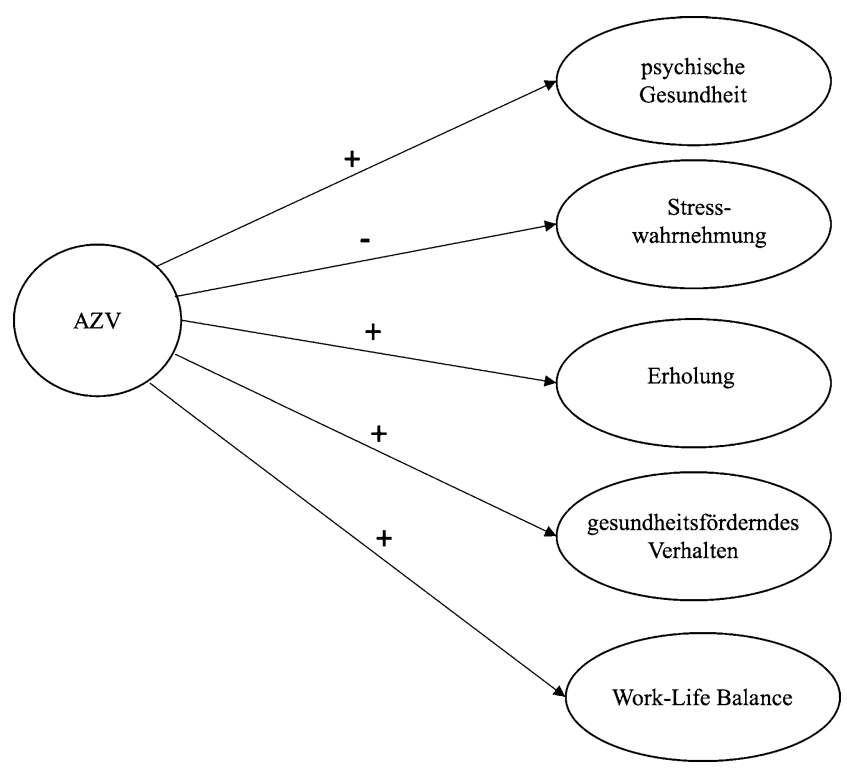

Abb. 4 Darstellung der Ergebnisse

für Freunde und Familie, was sich positiv auf die Vereinbarkeit von Familie und Beruf auswirkte. Außerdem war durch die Verkürzung der Arbeitszeit mehr Zeit für Erledigungen von Aufgaben im Haushalt.

\subsubsection{Erholung}

Auch die Möglichkeiten zur Erholung steigerten sich in fünf von fünf Studien. So wurde bspw. in den Fallstudien von Gerold et al. (2017) berichtet, dass die Steigerung der Freizeit für mehr Erholung sorgte. Zudem wurde ebenfalls eine verbesserte subjektive Schlafqualität verzeichnet (Åkerstedt et al. 2001; Schiller et al. 2017).

\subsubsection{Gesundheitsförderndes Verhalten}

AVZ wirkte sich bei zwei von drei Studien positiv auf das gesundheitsfördernde Verhalten der Teilnehmenden aus. Es zeigten sich in der Freizeit mehr Aktivitäten zur aktiven Erholung (Schiller et al. 2018) sowie eine Steigerung der Bewegung der Beschäftigten (Von Thiele Schwarz et al. 2008).

\subsubsection{Psychische Gesundheit}

In $80 \%$ der analysierten Studien hatte die AZV einen positiven Einfluss auf die psychische Gesundheit. Arbeitnehmende verspürten nach einer verringerten Arbeitszeit bei gleichbleibendem Lohnausgleich eine Verbesserung der geistigen Ermüdung (Åkerstedt et al. 2001; Barck-Holst et al. 2015) sowie des Wohlbefindens (Lepinteur 2019). Außerdem verbesserte sich die allgemeine innere Einstellung (Åkerstedt et al. 2001) und auch der Umgang mit negativen Emotionen (Barck-Holst et al. 2015).

\subsubsection{Stresswahrnehmung}

Ein Großteil (86\%) der Studien ergaben durch AZV positive Auswirkungen auf das Stressempfinden, Wohlbefinden und die Stresswahrnehmung. Bei der Erhebung von Åkerstedt et al. (2001) wurde bei der Umsetzung zusätzliches Personal eingestellt, wodurch sich das Arbeitspensum verringerte. Im Gegensatz dazu, zeigten dich bei der Studie von Von Thiele Schwarz et al. (2015), nach zwölf Monaten keine signifikante Verbesserung der Stresswahrnehmung, trotz der vergleichbaren Umsetzung der AZV. Bei Schiller et al. (2018) kam es trotz derselben Anzahl an Beschäftigten zu einer Verringerung des Arbeitspensums. In den Fallstudien von de Spiegelaere und Piasna (2017), gab es teilweise positive Auswirkungen, unter der Voraussetzung, dass die AZV vorab organisiert wurde und es zu keiner höheren Intensität der Arbeit kam.

Trotz der überwiegend positiven Resultate wurde bei einer Vielzahl von Organisationen die Arbeitszeit wieder auf die ursprüngliche zurückgestellt. Zwar wurde durch die AZV teilweise sogar eine Steigerung der Produktivität zu erkennen, allerdings war ein Hauptgrund für den Abbruch die betriebswirtschaftlichen Kosten durch die Umsetzung, zum Beispiel durch die Annahme von zusätzlichem Personal (Von Thiele Schwarz et al. 2015).

\section{Diskussion}

Das Ziel dieses systematischen Literatur Reviews war es den Stand der Forschung in Bezug auf die Auswirkungen von AZV auf die psychische Gesundheit zu beleuchten und somit Empfehlungen für die Umsetzung in Organisationen zu liefern. Ein Großteil der Studien verzeichnete einen positiven Effekt der AZV auf die Work-home Balance, Erholung, gesundheitsförderndes Verhalten, psychisches Gesundheit sowie Stresswahrnehmung. Dennoch wurde der Versuch der weiteren Umsetzung bei einer Vielzahl von Organisationen wieder abgebrochen. Es war zwar teilweise eine Steigerung der Produktivität zu erkennen, allerdings war der Hauptgrund für den Abbruch die betriebswirtschaftlichen Kosten durch die Umsetzung. Ein möglicher Erklärungsansatz könnte die Umsetzung von AZV sein. Dabei wurden drei Kategorien in der Implementierung erkannt:

I. AZV ohne Veränderung der Arbeitsprozesse bzw. des -pensums

II. AZV ohne Veränderung der Arbeitsprozesse, allerdings in Kombination mit zusätzlichem Personal 
III. AZV mit einer vorhergehenden Veränderung der Arbeitsprozesse, ohne zusätzliches Personal

Bei einer AZV ohne Veränderung der Arbeitsstrukturen (I.), könnte es auf Dauer zu einer Verdichtung von Arbeit kommen, da die vorhandene Arbeit in kürzerer Zeit erledigt werden muss. Dies könnte möglicherweise zu Lasten der Pausen gehen und nach dem Effort-Recovery Modell (Meijman und Mulder 1998) zufolge langfristig zu einer höheren Belastung während der Arbeitszeit führen. Die Variante II fängt diesen Effekt zwar auf, jedoch steigen durch die Einstellung zusätzlichen Personals die betriebswirtschaftlichen Kosten, was vor allem für Arbeitgeber wenig rentabel sein könnte. Möglicherweise könnte es auch zu einer qualitativen Veränderung von Arbeit kommen, wie veränderte Aufgaben-, Wissens- und soziale Merkmale (Morgeson und Humphrey 2006). Die dritte Variante wurde von De Spiegelaere und Piasna (2017) als erfolgreichste Umsetzungsmöglichkeit empfohlen. Diese scheint auf Dauer am ehesten zu einer Verringerung des Arbeitspensums und somit zu einer Verbesserung des Wohlbefindens und der Gesundheit zu führen. Gleichzeitig wäre diese Option auch für Arbeitgeber rentabel. Allerdings sind die Möglichkeiten der Optimierung von Arbeitsprozessen derzeit noch auf manche Branchen beschränkt, sodass es bis dato keine allgemeingültige Empfehlung ausgesprochen werden kann.

\subsection{Limitationen}

Durch die uneinheitliche Begriffsbenennung gab es Unterschiede in der Umsetzung von AZV. Die Bestimmung eines konkreten Maßes an Arbeitsstunden, welche zu einer verbesserten Gesundheit beiträgt, war anhand der vorhandenen Studien limitiert. Eine Generalisierung der Ergebnisse war aufgrund der geringen Stichprobenanzahl sowie der Einschränkung in den Branchen schwierig. Den Primärstudien war auch nicht eindeutig zu entnehmen, ob mit der Einführung der AZV möglicherweise andere, ebenfalls positiv wahrzunehmenden Initiativen der Unternehmen eingeführt wurden, wie etwa Job-Rotation als Maßnahme der Arbeitsorganisation oder etwa sozial-gesellschaftliche Maßnahmen (Paruzel et al. 2020).

\subsection{Empfehlung für zukünftige Forschung}

Da in den meisten deutschsprachigen Artikeln der Begriff Arbeitszeitverkürzung (AZV) und in den englischsprachigen Artikeln working time reduction (WTR) verwendet wurde, ist zu empfehlen diese Begriffe in Zukunft einheitlich zu gebrauchen. Durch die unterschiedlichen Operationalisierungen, sollten zukünftig mögliche Wirkmechanismen, Mediatoren und Moderatoren von AZV untersucht werden, um $\mathrm{zu}$ erforschen unter welchen Bedingungen
AZV sich positiv auf die psychische Gesundheit auswirkt, wie z.B. Flexibilisierung von Arbeitszeiten, die auch positive Auswirkungen auf die Gesundheit hat (Kauffeld et al. 2004). Zudem sollte analysiert werden ab welcher Reduzierung von Arbeitsstunden man von AZV sprechen kann und welches Maß an Arbeitsstunden sich positiv auf die psychische Gesundheit auswirkt. Zusätzlich sollte dabei angegeben und mit untersucht werden, ob andere Maßnahmen zusammen mit AZV eingesetzt wurden und welche Auswirkungen AZV auf soziale Prozesse im Unternehmen hat.

In den Primärstudien wurde häufig ein Längsschnitt-Design verwendet, welches die ersten zwölf bis 18 Monate der Umsetzung umfasste. Daher empfehlen wir zusätzlich Erhebungen in Organisationen mit und ohne Verkürzung der Arbeitszeit durchzuführen, um einen Vergleich herstellen zu können.

\subsection{Empfehlung für die Praxis}

Zwar kann bis dato noch keine allgemeingültige Empfehlung für die Umsetzung in die Praxis abgeleitet werden, allerdings wird für eine erfolgreiche Erprobung empfohlen, die Reduktion der Arbeitszeit vorab ausführlich zu planen und Optimierungsmöglichkeiten für die Ausführung von Tätigkeiten und Arbeitsprozessen zu identifizieren. Dabei sollte jedoch darauf geachtet werden, dass die psychologische Qualität der Arbeit nicht leidet. Tätigkeitsanalysen könnten maßgeblich dabei helfen. Bei der Reduzierung der Arbeitszeit sollten auch neue Technologien verstärkt genutzt werden, um Arbeitsvorgänge effizienter zu gestalten, wie beispielsweise, für die Nachbereitung von Besprechungen oder Workshops, eine App, die das Erstellen eines Protokolls erleichtert und versprechen, viel Arbeitszeit zu sparen. Zunächst sollte festgelegt werden welches Ziel erreicht werden soll und in welchem Ausmaß die Arbeitszeit reduziert werden soll. Abhängig von der Anzahl der Stunden, können die Wirkung und Konsequenzen unterschiedlich stark ausfallen (De Spiegelaere und Piasna 2017). Die Umsetzung kann zudem radikal oder linear erfolgen. Auf lange Sicht sollte die Verkürzung sowohl für den Arbeitgeber rentabel sein und zum anderen von Beschäftigen in der Ausführung der Arbeit umsetzbar sein. Um Beschäftigte nicht zu überfordern, sollten sie zudem bei der Veränderung der Arbeitsbedingungen mit einbezogen werden (Kauffeld und Maier 2020). In der Analyse wurden viele Erhebungen im Gesundheitswesen durchgeführt. Ein erfolgreiches Modell war beispielsweise die Umsetzung von Gyllensten et al. (2017). Hierbei wurde die Arbeitszeit verkürzt, auf Mittagspausen verzichtet und auch das Schichtsystem verändert. Das Schichtsystem wurde dabei von zwei auf drei Schichten angepasst, wodurch weder zusätzliches Personal eingestellt werden musste noch zusätzliche Kosten entstan- 
den. Bei der Umsetzung von AZV sollte außerdem darauf geachtet werden, dass diese kompatibel mit dem Arbeitsschutzgesetz in Deutschland sind, wie z.B. ausreichende Pausen je nach Umfang der Arbeitsstunden.

\subsection{Konklusion}

Erholung und mehr Freizeit ist vor allem in Zeiten höherer Arbeitsanforderungen unabdinglich, um die Gesundheit und Leistungsfähigkeit von Beschäftigung langfristig zu wahren. Bislang hat man sich bei der Optimierung von Gesundheit überwiegend auf Aufgabengestaltung und gesundheitsfördernde Aktivitäten gestützt. Die analysierten Studien weisen darauf hin, dass auch AZV bei gleichbleibendem Lohn eine Option sein könnte, um die Gesundheit zu verbessern. Dabei stellt die AZV eine Möglichkeit dar, um die Beanspruchungen zu reduzieren, sowie dem Wunsch der Beschäftigten nach mehr Freizeit nachzukommen. Voraussetzung dabei ist, dass es zu keiner höheren Intensität der Arbeit kommt. Dabei wird empfohlen die Reduktion der Arbeitszeit im Vorfeld detailliert vorzubereiten und Optimierungsmöglichkeiten für die Ausführung von Tätigkeiten und Arbeitsprozessen herauszustellen. Für eine allgemeingültige Empfehlung zur Umsetzung in die Praxis, bedarf es noch der systematischen Untersuchung dieser Gestaltungsmaßnahme im Vergleich zu anderen, mit denen vergleichbare Kriterien wie Gesundheit erreicht werden könnten.

Funding Open Access funding provided by Projekt DEAL.

Open Access Dieser Artikel wird unter der Creative Commons Namensnennung 4.0 International Lizenz veröffentlicht, welche die Nutzung, Vervielfältigung, Bearbeitung, Verbreitung und Wiedergabe in jeglichem Medium und Format erlaubt, sofern Sie den/die ursprünglichen Autor(en) und die Quelle ordnungsgemäß nennen, einen Link zur Creative Commons Lizenz beifügen und angeben, ob Änderungen vorgenommen wurden.

Die in diesem Artikel enthaltenen Bilder und sonstiges Drittmaterial unterliegen ebenfalls der genannten Creative Commons Lizenz, sofern sich aus der Abbildungslegende nichts anderes ergibt. Sofern das betreffende Material nicht unter der genannten Creative Commons Lizenz steht und die betreffende Handlung nicht nach gesetzlichen Vorschriften erlaubt ist, ist für die oben aufgeführten Weiterverwendungen des Materials die Einwilligung des jeweiligen Rechteinhabers einzuholen.

Weitere Details zur Lizenz entnehmen Sie bitte der Lizenzinformation auf http://creativecommons.org/licenses/by/4.0/deed.de.

\section{Literatur}

Åkerstedt, T., Olsson, B., Ingre, M., Holmgren, M., \& Kecklund, G. (2001). A 6-hour working day-effects on health and well-being. Journal of Human Ergology, 30(1-2), 197-202. https://doi.org/ 10.11183/jhe1972.30.197.

Anttila, T. (2005). Reduced working hours: Reshaping the duration, timing and tempo of work (Dissertation). University of Jyväskylä, Finland. http://urn.fi/URN:ISBN:951-39-2117-4
Ashford, N., \& Kallis, G. (2013). A four-day workweek: a policy for improving employment and environmental conditions in Europe. The European Financial Review. 30. April 2013

Bannai, A., \& Tamakoshi, A. (2014). The association between long working hours and health: a systematic review of epidemiological evidence. Scandinavian Journal of Work, Environment, and Health, 40(1), 5-18. https://doi.org/10.5271/sjweh.3388.

Barck-Holst, P., Nilsonne, A., Åkerstedt, T., \& Hellgren, C. (2015). Reduced working hours and stress in the Swedish social services: a longitudinal study. International Social Work, 60(4), 897-913. https://doi.org/10.1177/0020872815580045.

Barck-Holst, P., Nilsonne, A.., Åkerstedt, T., \& Hellgren, C. (2019). Coping with stressful situations in social work before and after reduced working hours, a mixed-methods study. European Journal of Social Work, 5(1), 1-15. https://doi.org/10.1080/13691457. 2019.1656171.

Barnett, R.C. (2006). Relationship of the number and distribution of work hours to health and quality-of-life (QOL) outcomes. In P.L. Perrewe \& D.C. Ganster (Hrsg.), Research in occupational stress and well-being, employee health, coping, and methodologies (S. 99-138). Amsterdam: Elsevier.

Bärschneider, N. (2020). Kürzer, besser, glücklicher. Frankfurter Allgemeine Zeitung. https://www.faz.net/aktuell/karriere-hochschule/ buero-co/sechs-stunden-arbeitstag-kann-das-gutgehen- 16565666. html (Erstellt: 7. Jan. 2020). Zugegriffen: 31. Jan. 2020.

Böckmann, C. (2018). IG Metall setzt Arbeitszeitverkürzung durch. vdi Nachrichten. https://www.vdi-nachrichten.com/Gesellschaft/ IG-Metall-setzt-Arbeitszeitverkuerzung (Erstellt: 27. Juni 2018). Zugegriffen: 31. Jan. 2020.

Brodsky, A., \& Amabile, T. M. (2018). The downside of downtime: the prevalence and work pacing consequences of idle time at work. Journal of Applied Psychology, 103(5), 496-512. https://doi.org/ 10.1037/apl0000294.

Davis, J., Mengersen, K., Bennett, S. \& Mazerolle, L. (Hrsg.). (2014). Viewing systematic reviews and meta-analysis in social research through different lenses. SpringerPlus. https://doi.org/10.1186/ 2193-1801-3-511

Deci, E. L., \& Ryan, R. M. (2008). Self-determination theory: a macrotheory of human motivation, development, and health. Canadian Psychology/Psychology randomized, 49(3), 182-185. https://doi. org/10.1037/a0012801.

De Spiegelaere, S., \& Piasna, A. (2017). The why and the how of working time reduction. Brüssel: European Trade Union Institute.

Diewald, M., Andernach, B., \& Kunze, E. S. (2020). Entwicklung der Beschäftigungsstruktur durch Digitalisierung von Arbeit. In G. Maier, G. Engels \& E. Steffen (Hrsg.), Handbuch Gestaltung digitaler und vernetzter Arbeitswelten (S. 5). Berlin Heidelberg: Springer.

Dougall, M.D. (2020). How Finland's fake four-day week became a 'fact' in Europe's media News now Finland. https:// newsnowfinland.fi/politics/how-finlands-fake-four-day-weekbecame-a-fact-in-europes-media (Erstellt: 6. Jan. 2020). Zugegriffen: 31. Jan. 2020.

Frankfurter Allgemeine Zeitung (Hrsg.). (2019). Lieber mehr Freizeit als mehr Geld. https://www.faz.net/aktuell/karriere-hochschule/ buero-co/gewerkschafts-umfrage-lieber-mehr-freizeit-als-mehrgeld-16437447.html (Erstellt: 17. Okt. 2019). Zugegriffen: 18. Dez. 2019.

Frey, C.B., \& Osborne, M.A. (2017). The future of employment. How susceptible are jobs to computerization? Technological Forecasting and Social Change, 114(1), 254-280. https://doi.org/ 10.1016/j.techfore.2016.08.019.

Ganster, D.C., Rosen, C.C., \& Fisher, G.G. (2018). Long working hours and well-being: what we know, what we do not know, and what we need to know. Journal of Business and Psychology, 33(1), 25-39. https://doi.org/10.1007/s10869-016-9478-1. 
Gerold, S., Soder, M., \& Schwendinger, M. (2017). Arbeitszeitverkürzung in der Praxis. Innovative Modelle in österreichischen Betrieben. Wirtschaft und Gesellschaft, 43(2), 177-204.

Grimm, K. (2017). Warum der 6-Stunden-Arbeitstag in Schweden nicht funktioniert. Stern. https://www.stern.de/wirtschaft/job/6stunden-arbeitstag--schweden-stampft-testprojekt-ein-7268662. html (Erstellt: 8. Jan. 2017). Zugegriffen: 31. Jan. 2020.

Gyllensten, K., Andersson, G., \& Muller, H. (2017). Experiences of reduced work hours for nurses and assistant nurses at a surgical department: a qualitative study. BMC Nursing, 16(1), 16. https:// doi.org/10.1186/s12912-017-0210-x.

Hackman, J., \& Oldham, G. (1976). Motivation through the design of work: test of a theory. Organizational Behavior and Human Performance, 16, 250-279. https://doi.org/10.1016/00305073(76)90016-7.

Jacobs, L. (2018). Wollt ihr weniger arbeiten und genauso viel verdienen?. Die Zeit. https://www.zeit.de/zeit-spezial/2018/01/25stunden-woche-lasse-rheingans-agentur-bielefeld (Erstellt: 10. Apr. 2018). Zugegriffen: 7. Jan. 2020.

Kaiser, D. (2018). Tarifeinigung mit EVG steht - höhere Löhne und mehr Wahlmodelle - gute Botschaft für Kunden. Deutsche Bahn. https://www.deutschebahn.com/de/presse/pressestart_zentrales_ uebersicht/Tarifeinigung-mit-EVG-steht-hoehere-Loehne-undmehr-Wahlmodelle-gute-Botschaft-fuer-Kunden-3575420 (Erstellt: 15. Dez. 2018). Zugegriffen: 16. Juli 2019.

Kang, M.-Y., Park, H., Seo, J.-C., Lim, D., Lim, Y.-H., Lim, S., Cho, S.H., \& Hong, Y.-C. (2012). Long working hours and cardiovascular disease. Journal of Occupational and Environmental Medicine, 54, 532-537. https://doi.org/10.1097/JOM. 0b013e31824fe192.

Kauffeld, S., \& Maier, G. W. (2020). Digitalisierte Arbeitswelt. Gruppe. Interaktion. Organisation, 51, 1-4. https://doi.org/10.1007/ s11612-020-00508-y.

Kauffeld, S., Jonas, E., \& Frey, D. (2004). Effects of flexible work-time design on employee- and company-related aims. European Journal of Work and Organizational Psychology, 13, 79-100. https:// doi.org/10.1080/13594320444000001.

Lepinteur, A. (2019). The shorter workweek and worker wellbeing: evidence from Portugal and France. Labour Economics, 58(1), 204-220. https://doi.org/10.1016/j.labeco.2018.05.010.

Lohmann-Haislah, A. (2012). Stressreport Deutschland 2012. Dortmund: Bundesanstalt für Arbeitsschutz und Arbeitsmedizin. http://www.baua.de/de/Publikationen/Fachbeitraege/Gd68.pdf? blob=publicationFile

Luftman, J., Lyytinen, K., \& ben Zvi, T. (2017). Enhancing the measurement of information technology (IT) business alignment and its influence on company performance. Journal of Information Technology, 32(1), 26-46. https://doi.org/10.1057/jit.2015.23.

MacInnes, J. (2005). Work-life balance and the demand for reduction in working hours: evidence from the British social attitudes survey 2002. British Journal of Industrial Relations, 43(2), 273-295. https://doi.org/10.1111/j.1467-8543.2005.00355.x.

Meijman, T.F., \& Mulder, G. (1998). Psychological aspects of workload In P. J.D. Drenth \& H. Thierry (Hrsg.), Handbook of work and organizational psychology: work psychology (2. Aufl. S. 5-33). Hove: Psychology Press.

Moher, D., Liberati, A., Tetzlaff, J., \& Altman, D. G. (2009). Preferred reporting items for systematic reviews and meta-analyses: The PRISMA statement. Annals of Internal Medicine, 151(4), 264-269. https://doi.org/10.7326/0003-4819-151-4-20090818000135.

Morgeson, F. P., \& Humphrey, S.E. (2006). The Work Design Questionnaire (WDQ): developing and validating a comprehensive measure for assessing job design and the nature of work. Journal of Applied Psychology, 91(6), 1321-1339. https://doi.org/10. 1037/0021-9010.91.6.1321.
Newport, C. (2019). 5-Hour Workdays? 4-Day Workweeks? Yes, Please. New York Times. https://www.nytimes.com/2019/11/06/ opinion/five-hour-workday-experiment.html (Erstellt: 6. Nov. 2019). Zuggeriffen: 18. Dez. 2019.

Parent-Thirion, A., Biletta, I., Cabrita, J., Vargas, O., Vermeylen, G., Wilczynska, A., \& Wilkens, M. (2016). Sixth European working conditions survey: overview report. Luxemburg: European Foundation for the Improvement of Living and Working Conditions. https://www.eurofound.europa.eu/publications/report/2016/ working-conditions/sixth-european-working-conditions-surveyoverview-report.

Parkinson, C. N., \& Osborn, R.C. (1957). Parkinson's law, and other studies in administration (24. Aufl.). Boston: Houghton Mifflin.

Paruzel, A., Klug, H., \& Maier, G. W. (2020). The relationship between perceived corporate social responsibility and employee-related attitudes and behavior: a meta-analysis. Manuscript submitted for publication.

Schaff, A. (2019). Arbeit 4.0: Risiken für die psychische Gesundheit. In B. Hermeier, T. Heupel \& S. Fichtner-Rosada (Hrsg.), Arbeitswelten der Zukunft. Berlin Heidelberg: Springer. https://doi.org/ 10.1007/978-3-658-23397-6_17.

Schiller, H., Lekander, M., Rajaleid, K., Hellgren, C., Åkerstedt, T., Barck-Holst, P., \& Kecklund, G. (2017). The impact of reduced worktime on sleep and perceived stress-a group randomized intervention study using diary data. Scandinavian Journal of Work, Environment \& Health, 43(2), 109-116.

Schiller, H., Lekander, M., Rajaleid, K., Hellgren, C., Åkerstedt, T., Barck-Holst, P., \& Kecklund, G. (2018). Total workload and recovery in relation to worktime reduction: a randomized controlled intervention study with time-use data. Occupational and Environmental Medicine, 75(3), 218-226. https://doi.org/10.1136/oemed2017-104592.

Solovieva, S., Lallluka, T., Virtanen, M., \& Viikar-Juntura, E. (2013). Psychosocial factors at work, long work hours, and obesity: a systematic review. Scandinavian Journal of Work, Environment, and Health, 39(1), 241-258. https://doi.org/10.5271/sjweh.3364.

Sonnentag, S. (2001). Work, recovery activities, and individual wellbeing: a diary study. Journal of Occupational Health Psychology, 6(3), 196-210. https://doi.org/10.1037/1076-8998.6.3.196.

Sonnentag, S., Binnewies, C., \& Mojza, E. J. (2010). Staying well and engaged when demands are high: The role of psychological detachment. Journal of Applied Psychology, 95(1), 965-976. https:// doi.org/10.1037/a0020032.

Sparks, K., Cooper, C., Fried, Y., \& Shirom, A. (1997). The effects of hours of work on health: a meta-analytic review. Journal of Occupational and Organizational Psychology, 70, 391-408. https:// doi.org/10.1111/j.2044-8325.1997.tb00656.x.

Thörel, E., Pauls, N., \& Göritz, A. S. (2020). Antezedenzien und Wirkmechanismen arbeitsbezogener erweiterter Erreichbarkeit - Wenn die Arbeitszeit nicht ausreicht. Gruppe. Interaktion. Organisation, 51, 37-47. https://doi.org/10.1007/s11612-020-00496-z.

Trougakos, J.P., Beal, D. J., Green, S. G., \& Weiss, H. M. (2008). Making the break count: an episodic examination of recovery activities, emotional experiences, and positive affective displays. Academy of Management Journal, 51(1), 131-146. https://doi.org/10. 5465/amj.2008.30764063.

Von Thiele Schwarz, U., Hasson, H., \& Lindfors, P. (2015). Derailed or failed? A closer look at reduced working hours as an occupational health intervention. In M. Karanika-Murray \& C. Biron (Hrsg.), Derailed organizational interventions for stress and well-being (S. 21-26). Berlin Heidelberg: Springer.

Von Thiele Schwarz, U., Lindfors, P., \& Lundberg, U. (2008). Healthrelated effects of worksite interventions involving physical exercise and reduced workhours. Scandinavian Journal of Work, Environment and Health, 34(3), 179-188. https://doi.org/10.5271/ sjweh.1227. 
Webber, A. (2019). Should we rethink our working hours to boost productivity?. Personnel Today. https://www.personneltoday.com/ hr/should-we-rethink-our-working-hours-to-boost-productivity/ (Erstellt: 17. Apr. 2019). Zuggeriffen: 18. Juli 2019.

Wöhrmann, A.M., Gerstenberg, S., Hünefeld, L., Pundt, F., Reeske-Behrens, A., Brenscheidt, F., \& Beermann, B. (2016). Arbeitszeitreport Deutschland 2016. Dortmund: Bundesanstalt für Arbeitsschutz und Arbeitsmedizin. https://www.baua.de/DE/ Angebote/Publikationen/Berichte/F2398.pdf?_blob=publication File.



Virgilia V. Jansen-Preilowski ist selbstständige Trainerin und systemischer Coach. Sie promoviert extern am Lehrstuhl für Arbeits- und Organisationspsychologie der Universität Bielefeld. Ihre Forschungsinteressen liegen in flexiblen $\mathrm{Ar}$ beitszeitmodellen sowie Auswirkungen digitalisierter Arbeit auf Beschäftigte.

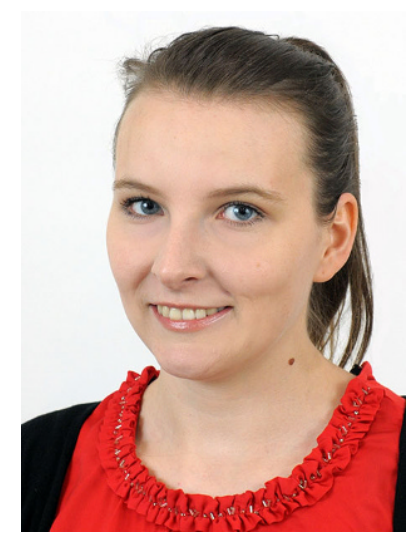

Agnieszka Paruzel ist wissenschaftliche Mitarbeiterin am Lehrstuhl für Arbeits- und Organisationspsychologie der Universität Bielefeld. Ihre Forschungsinteressen liegen in den Bereichen Corporate Social Responsibility, Arbeitgeberattraktivität sowie Auswirkungen digitalisierter Arbeit auf Beschäftigte.

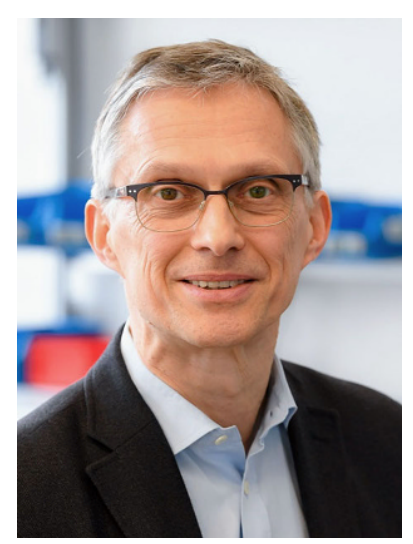

Prof. Dr. Günter W. Maier ist Professor am Lehrstuhl für Arbeitsund Organisationspsychologie der Universität Bielefeld sowie stellvertretender Sprecher des Forschungsschwerpunkts „Digitale Zukunft“ und des NRW Forschungskollegs „Gestaltung von flexiblen Arbeitswelten - Menschen-zentrierte Nutzung von Cyber-Physical Systems in Industrie 4.0“. Seine Forschungsinteressen liegen in den Bereichen Gerechtigkeit in der Mensch-Maschine-Interaktion, Qualifizierungsbedarf, Führung und Gestaltung von Arbeit in der digitalen Transformation sowie Kreativität. 